\title{
Cannabinoid Receptor CB2 Subtype: A Novel Pharmacological Target in Cancer and Neurodegenerative Diseases
}

\section{Contino $\mathbf{M}^{*}$ and Giampietro R}

Dipartimento di Farmacia-Scienze del Farmaco, Università degli Studi di Bari Aldo Moro, Italy

${ }^{*}$ Correscponding author: Contino M, Dipartimento di Farmacia-Scienze del

\section{Editorial}

Volume 3 Issue 2

Received Date: June 03, 2019

Published Date: June 07, 2019

DOI: $10.23880 /$ oajpr-16000178

Farmaco, Università degli Studi di Bari ALDO MORO, via Orabona 4, 70124, Bari, Italy, Tel: +390805442747; Email: marialessandra.contino@uniba.it

Keywords: Cannabinoid receptor; Neurodegenerative diseases; Cancer

Abbreviations: CB2R: CB2 Receptor; ECS: Endocannabinoid System; GPCRs: G-Protein Coupled Receptors; AC: Adenyl Cyclase; PLC: Phospholipase C; CNS: Central Nervous System; AD: Alzheimers Disease, PD: Parkinsons Disease, HD: Huntington's Disease, MS: Multiple Sclerosis; SLA: Sclerosis Lateral Amyotrophic; SAR: Structure Activity Relationship.

\section{Editorial}

CB2 receptor (CB2R) is one of the canonical cannabinoid receptor belonging to endocannabinoid system (ECS). It was cloned for the first time in 1993 from the human leukemia cell line HL-60, and it is part of the Gprotein coupled receptors family (GPCRs), being coupled to a Gi/o protein [1-3]. CB2R triggers diverse signaling pathways depending on the type of cells and tissues where it is expressed. CB2R signaling mechanisms encompasses: 1) the inhibition of Adenyl ciclase (AC); 2) MAPK activation; 3) the stimulation of phospholipase $C$ (PLC) and inositol 1,4,5-triphosphate (IP3) with an increase of intracellular calcium concentration [4,5]. Thus, depending on the environment cellular circumstances, CB2R engagement can activate several signaling pathways in different ways [4-6]. CB2R is defined as "peripheral receptor for cannabinoids" because of its primarily distribution in immune cells; CB2R mRNA was found in the spleen, tonsils, thymus, mast cells, blood cells and bone marrow $[3,5]$. Recent studies have detected CB2R also in the central nervous system (CNS), mainly in the active-microglia, involved in neuroinflammation response and an overexpression of the receptors has been found in different types of tumours [7,8]. Therefore, based on these data, nowadays CB2R is considered as a novel target for the development of pharmacological approaches in the treatment of various illnesses, as cancer and neurodegenerative diseases $[5,9]$.

In the oncological field, as previously reported, a correlation between the overexpression of CB2R and diverse tumors, as prostate cancer, lymphoma, breast cancer, acute myeloid leukemia, hepatocellular carcinoma has been observed [7]. Although some studies assert that the loss of expression of cannabinoid receptors can also enhance the tumor growth, evidences suggest that the degree of upregulation of CB2R levels corresponds to tumor aggression and progression $[5,10]$. Nevertheless, several studies have evaluated the antitumor effect of different types of CB2R molecules already used in cancer therapy for their well-established palliative effects. Several works have underlined that endogenous, phytogenic, synthetic cannabinoids, and modulators of endocannabinoid biosynthesis, are able to inhibit the proliferation of a wide spectrum of tumor cells with mechanism not totally unravelled [11]. Velasco, et al. suggested at least four anticancer mechanisms: 1) the suppression of mitogenic signal; 2) the induction of apoptosis; 3) the inhibition of tumor angiogenesis; 4) induction of metastasis [11]. Concerning to CB2R agonists, among phytocannabinoid, morin, a flavonoid structurally closely related to quercetin, showed an apoptotic effect by 


\section{Open Access Journal of Pharmaceutical Research}

a mechanism not fully resolved [10]. Interestingly, synthetic compounds as WIN55,212-2, JWH-015, JWH133 were found able to induce cannabinoid receptor dependent anti-proliferative and pro-apoptotic effects both in vitro and in vivo assays [7,11-15]. Recently, researchers have discovered the ability of $\mathrm{CB} 2 \mathrm{R}$ to form heteromers with other GPCRs, as CXCR4 or GPR55. For instance, in human breast and prostate cancer cells, the $\mathrm{G}$ protein-coupled chemokine receptor CXCR4, physiologically responsible for cell proliferation and migration, associated to $\mathrm{CB} 2 \mathrm{R}$ yields the functional heteromers CXCR4-CB2R, which leads to a decrease of cancer spread [14].

In the neuroinflammation field, the connection between CB2R and neurodegenerative diseases is due to the identified CB2R up-regulation in specific CNS areas, notably in glial cells, as microglia, the resident immune cells of the brain involved in case of pathological damage. In detail, different studies widely demonstrated that CB2R up-regulation on activated microglial cells is the first step in neurodegeneration [17]. As studied by Mecha, et al. under specific injury stimuli within the brain, microglia cells can switch from M1 (neurotoxic state) to M2 (neuroprotective state) state restoring normal tissue homeostasis. A pivotal role in this transition is played by CB2R, overexpressed on microglia cells under neuroinflammatory conditions [16]. The activation of CB2R on microglial cells inhibits the release of proinflammatory cytokines and triggers the release of antiinflammatory molecules, controlling the processes of proliferation, migration and differentiation of these cells [17]. This neuroinflammatory state is common in neurodegenerative diseases as like Alzheimer's disease (AD), Parkinson's disease (PD), Huntington's disease (HD), multiple sclerosis (MS) and sclerosis lateral amyotrophic (SLA).

CB2R agonists and inverse agonists have emerged as neuroprotective agents. With this in mind, structureactivity relationship (SAR) and structure-affinity relationship (SAfiR) studies have been performed on different scaffolds with the aim to identify the molecular features useful for the design of both therapeutic and diagnostic agents for these neurodegenerative disorders [18]. Experiments have shown that unlike WIN55, 212-2, JWH-133 is able to reduce inflammatory reactions associated to fibrillar b-amyloid $(\mathrm{A} \beta)$ plaques production and deposition, characterizing AD $[8,19]$. Further studies suggested that even in this disorder, the activation of CB2R by JWH-133 and WIN55, 212-2 facilitates $\mathrm{A} \beta$ clearance [19].
Diverse studies have underlined that also microglia cells in PD patients overexpressed CB2R, awarding CB2R agonists pivotal for anti-oxidant and neuroprotective effects they can induce [8].

Recently, $\mathrm{Bu}$ et al., have demonstrated the neuroprotection ability of a CB2R inverse agonist, SMM189 , adept in converting microglia cells in M2 state, rescuing neurons in cortex, striatum and amygdala [20].

Finally, bearing in mind the pleiotropic activity of CB2R and the lack of undesirable psychoactive effects after its activation (differently from the other subtype CB1R activation), we can assert that CB2R represents a promising pharmacological target in cancer and in neurodegenerative disorders.

\section{References}

1. Petwee RG (2006) Cannabinoid pharmacology: the first 66 years. British Journal of Pharmacology 147: S163-S171.

2. Grotenhermen F (2003) Pharmacokinetics and Pharmacodynamics of Cannabinoids. Clin Pharmacokinet 42(4): 327-360.

3. Svìzenskà I, Dubový P, Sulcovà A (2008) Cannabinoid receptors 1 and 2 (CB1 and CB2), their distribution, ligands and functional involvement in nervous system stuctures - A short review. Pharmacology, Biochemistry and Behavior 90(4): 501-511.

4. Dhopeshwarkar A, Mackie K (2014) CB2 Cannabinoid Receptors as a Therapeutic Target - What Does the Future Hold?. Mol Pharmacol 86(4): 430-437.

5. Tabrizi MA, Baraldi PG, Borea PA, Varani K (2016) Medicinal Chemistry, Pharmacology, and Potential Therapeutic Benefits of Cannabinoid CB2 Receptor Agonists. Chem Rev 116(2): 519-560.

6. Malfitano AM, Basu S, Maresz K, Bifulco M, Dittel BN (2014) What We Know and Don't Know About the Cannabinoid Receptor 2 (CB2). Semin Immunol 26(5): 369-379.

7. Chakravarti B, Ravi J, Ganju RK (2014) Cannabinoids as therapeutic agents in cancer: current status and future implications. Oncotarget 5(15): 5852-5872.

8. Cassano T, Calcagnini S, Pace L, De Marco F, Romano A, et al. (2017) Cannabinoid receptor-2 signaling in neurodegenerative disorders: from pathogenesis to a 


\section{Open Access Journal of Pharmaceutical Research}

promising therapeutic target. Frontiers in Neuroscience 11: 30.

9. Chen D, Gao M, Gao F, Su Q, Wu J (2017) Brain Cannabinoid Receptor 2: expression, function and modulation. Acta Pharmacologica Sinica 38(3): 313316.

10. Wang D, Wang H, Ning W, Backlund MG, Dey SK (2008) Loss of cannabinoid receptor 1 accelerates intestinal tumor growth. Cancer Res 68(15): 64686476.

11. Morales P, Jagerovic N (2019) Antitumor Cannabinoid Chemotypes: Structural Insights. Front. Pharmacol 10: 621 .

12. Velasco G, Sánchez C, Guzmán M (2016) Anticancer mechanisms of cannabinoids. Curr Oncol 23(2): S23S32.

13. Pisanti S, Malfitano AM, Grimaldi C, Santoro A, Gazzerro P, et al. (2009) Use of cannabinoid receptor agonists in cancer therapy as palliative and curative agents. Best Practice \& Research Clinical Endocrinology \& Metabolism 23(2): 117-131.

14. Moreno E, Cavic M, Krikokuca A, Casadò V, Canela E (2019) The endocannabinoid system as a target in cancer disease: are we there yet?. Front Pharmacol 10: 339 .
15. Alexander A, Smith PF, Rosengren RJ (2009) Cannabinoids in the treatment of cancer. Cancer Letter 285: 6-12.

16. Mecha $\mathrm{M}$, Feliù $\mathrm{A}$, Carrillo-Salinas FJ, Rueda-Zubiaurre A, Ortega-Gutièrrez $S$, et al. (2015) Endocannabinoids drive the acquisition of an alternative phenotype in microglia. Brain Behav Immun 49: 233-245.

17. Fernàndez-Ruiz J, Pazos $M R$, Garc'ia-Arencibia $M$, Sagredo 0, Ramos JA (2008) Role of CB2 receptor in neuroprotective effects of cannabinoids. Molecular and Cellular Endocrinology 286(1): S91-S96.

18. Spinelli F, Capparelli E, Abate C, Colabufo NA, Contino A (2017) Perspectives of cannabinoid type 2 receptor (CB2R) ligand in neurodegenerative disorders: structure Affinity relationship (SAfiR) and structure activity relationship (SAR) studies. J Med Chem 60(24): 9913-9931.

19. Aso E, Ferrer I (2016) CB2 cannabinoid receptor as potential target against Alzheimer's disease. Front Neurosci 10: 243.

20. Bu W, Ren H, Deng Y, Del Mar N, Guley NM, et al. (2016) Mild Traumatic Brain Injury Produces Neuron Loss That Can Be Rescued by Modulating Microglial Activation Using a CB2 Receptor Inverse Agonist Front. Neurosci 10: 449. 